\title{
KÇ3B-ESA: Hiperspektral Görüntü Sınıflandırması için Yeni 3B Evrişimli Sinir Ağı ve Uzaktan Algılama Uygulaması
}

\author{
Mücahit Cihan $^{1 *}$, Murat Ceylan ${ }^{2}$ \\ ${ }^{1}$ Konya Teknik Üniversitesi, Mühendislik ve Doğa Bilimleri Fakültesi, Elektrik-Elektronik Mühendisliği Bölümü, Konya, Türkiye (ORCID: 0000-0002-1426-319X) \\ ${ }^{2}$ Konya Teknik Üniversitesi, Mühendislik ve Doğa Bilimleri Fakültesi, Elektrik-Elektronik Mühendisliği Bölümü, Konya, Türkiye (ORCID: 0000-0001-6503-9668)
}

( $1^{\text {st }}$ International Conference on Computer, Electrical and Electronic Sciences ICCEES 2020 - 8-10 Ekim 2020)

(DOI: $10.31590 /$ ejosat.802890)

ATIF/REFERENCE: Cihan, M. \& Ceylan, M. (2020). KÇ3B-ESA: Hiperspektral Görüntü Sınıflandırması için Yeni 3B Evrişimli Sinir Ağı ve Uzaktan Algılama Uygulaması. Avrupa Bilim ve Teknoloji Dergisi, (Özel Say1), 65-71.

$\ddot{O} \mathbf{z}$

Hiperspektral Görüntüleme (HSG) uzamsal ve spektral bilgiyi içeren yüzlerce banttan oluşur. HSG verileri sınıflandırılırken uzamsal özelliklerin yanında spektral özelliklerinde elde edilmesi büyük önem taşır. Bu çalışmada hem uzamsal hem de spektral bilgilerin elde edilmesi için yeni bir derin öğrenme modeli önerilmiştir. Öncelikle, HSG verilerinin boyutlarının büyük olmasından dolayı tüm verilere Temel Bileşen Analizi (TBA) uygulanarak uzamsal boyut değişmeyecek şekilde spektral boyut küçültülmüştür. Daha sonra yeni bir yöntem olan, HSG verilerinin sınıflandırıldığı çalışmalarda yer alan, Komşuluk Çıkarımı (KÇ) yöntemi kullanılmıştır. Bu yöntem ile tüm pikselleri tarayacak şekilde mini küpler oluşturularak örnek sayısı artırılmıştır. Son olarak oluşturulan bu küpler 3B konvolüsyon katmanlarının bulunduğu 3B-Evrişimli Sinir Ağı (3B-ESA) modeli ile eğitilmiştir. Bu sayede daha anlamlı özelliklerin elde edilmesi sağlanmıştır. Önerilen modeli test etmek için Indian Pines (IP), Salinas Scene (SA) ve Pavia University (PU) uzaktan algılama veri setleri kullanılarak HSG sınıflandırma deneyleri yürütülmüştür. Yürütülen bu deneyler sonucunda tüm veri setleri için genel doğruluk (GD), kappa katsayısı (KC) ve ortalama doğruluk (OD) değerleri hesaplanarak sınıflandırma performansı değerlendirilmiştir. Sınıflandırma işlemi sonucunda IP veri seti için $\% 99.10 \mathrm{GD}$, \%98.97 KC, \%96.23 OD; SA veri seti için \%100 GD, \%100 KC, \%100 OD; ve son olarak PU veri seti için \%99.90 GD, \%99.87 KC, \%99.67 OD doğruluk oranları elde edilmiştir. Daha sonra bu sonuçlar gelişmiş derin öğrenme tabanlı metotlarla karşılaştırılarak, önerilen KÇ3B-ESA modelinin çok daha iyi bir performans gösterdiği kanıtlanmıştır.

Anahtar Kelimeler: Hiperspektral Görüntü Sınıflandırma, Derin Öğrenme, 3B-ESA, Komşuluk Çıkarımı, Uzamsal-spektral Özellikler, KÇ3B-ESA.

\section{NE3D-CNN: A New 3D Convolutional Neural Network for Hyperspectral Image Classification and Remote Sensing Application}

\begin{abstract}
Hyperspectral Imaging (HSI) consists of hundreds of bands containing spatial and spectral information. When classifying HSI data, it is of great importance to obtain spectral features as well as spatial features. In this study, a new deep learning model is proposed to obtain both spatial and spectral information. First of all, due to the large size of HSI data, Principal Component Analysis (PCA) was applied to all data and the spectral size was reduced so that the spatial dimension would not change. Then, Neighbourhood Extraction (NE) method, which is a new method used in studies in which HSI data were classified, was used. With the method, the number of samples was increased by creating mini cubes to scan all pixels. Finally, the cubes were trained with the 3D-Convolutional Neural
\end{abstract}

* Sorumlu Yazar: Konya Teknik Üniversitesi, Mühendislik ve Doğa Bilimleri Fakültesi, Elektrik-Elektronik Mühendisliği Bölümü, Konya, Türkiye, ORCID: 0000-0002-1426-319X, mcihan@ktun.edu.tr 
Network (3D-CNN) model, which has 3D convolution layers. In this way, more meaningful features were obtained. HSI classification experiments were conducted using Indian Pines (IP), Salinas Scene (SA) and Pavia University (PU) remote sensing datasets to test the proposed model. As a result of the experiments, the classification performance was evaluated by calculating the overall accuracy (OA), kappa coefficient (KC) and average accuracy (AA) values for all data sets. At the end of the classification process, accuracy rates of $99.10 \% \mathrm{OA}, 98.97 \% \mathrm{KC}, 96.23 \%$ AA for the IP data set, $100 \% \mathrm{OA}, 100 \% \mathrm{KC}, 100 \%$ AA for SA data set, and finally $99.90 \%$ OA, $99.87 \%$ KC, $99.67 \%$ AA for the PU data set were obtained. Later, by comparing the results with state-of-the-art deep learningbased methods, it has been proven that the proposed NE3D-CNN model gives a much better performance.

Keywords: Hyperspectral Image Classification, Deep Learning, 3D-CNN, Neighborhood Extraction, Spatial-spectral Features, NE3D-CNN.

\section{Giriş}

Hiperspektral Görüntüleme (HSG), yüzey materyallerinden yansıyan enerjinin, dar ve bitişik çok sayıda dalga boyu bandında ölçümüdür. Hiperspektral sensörler kullanılarak elde edilen veriler ilk iki boyut uzamsal üçüncü boyut spektral bilgiyi içeren hacimsel verilerdir. Ayrıca her bir piksel yüksek boyutlu vektörlerden oluşmaktadır. Elektromanyetik spektrum bandında hem görünür hem de kızılötesi bölgesinde birçok dalga boyunda görüntüler edebilen HSG, daha az sayıda görüntüler elde edebilen multispektral görüntülemeye göre daha anlamlı özellikler içermektedir. Bu özellikler farklı nesneleri tespit etmede yardımcı olabilir. HSG kullanılarak nesnelerin tespiti ve sınıflandırılması gibi son zamanlardaki yapılan çalışmalar, bağlamsal özelliklerin büyük avantajlar sağlayabileceğini göstermiştir (Lee ve Kwon, 2016; Chu ve ark., 2018).

HSG verilerinin etkili ve doğru bir şekilde sınıflandırılması için farklı algoritmalar sıklıkla kullanılmıştır. Melgani ve Buruzzone (2004), çok sınıflı problemleri çözmek için Destek Vektör Makinesi (DVM) algoritmasını kullanılmışlardır. Camps-Valls ve Buruzzone (2005), kernel tabanlı metotlar kullanarak DVM algoritması ile hiperspektral verileri sınıflandırmışlardır. Ayrıca farklı algoritmalar kullanılarak HSG verilerinin sınıflandırıldığı birçok çalışma mevcuttur. Örneğin; Rastgele Orman (RO) (Ham ve ark., 2005; Lawrence ve ark., 2006), K-En Yakın Komşu (K-Nearest Neighbors, KNN) (Ma ve ark., 2010; Huang ve ark., 2016) vb. gibi. Ama bu yöntemler kullanıldığında, özellik çıkarma işlemi uzun sürmekte ve önemli özelliklerin kaybedilmesi mümkün olmaktadır.

Derin öğrenme metotlarından birisi olan Evrişimli Sinir Ağı (ESA) nesne algılama (Ren ve ark., 2015), görüntü sınıflandırma (Wang ve ark., 2016), derinlik tahmini (Liu ve ark., 2015), anlamsal bölümleme (Gidaris ve Komodakis, 2015), cilt kanseri sınıflandırması (Saba ve ark., 2019) gibi alanlarda yüksek başarımlara sahiptir. Bunun sebebi, ESA'nın çok fazla ön işleme olmadan ağda bulunan gizli katmanlar ile özellikleri çıkarabilmesidir. Son yıllarda, bazı araştırmacılar hiperspektral verileri sınıflandırmak için farklı teknikler kullanmışlardır. Zhao ve Du (2016), HSG sınıflandırma için boyut indirgeme ve derin öğrenme teknikleri ile uzamsalspektral özellikleri çıkarmışlardır. Li ve ark. (2017), uzamsal özellikleri geliştirmek için derin ESA tabanlı HSG yeniden yapılanma modeli geliştirmişlerdir. Bir başka çalışmada Zhong ve ark. (2017), HSG sınıflandırma için uzamsal-spektral residual network (SSRN) amaçlamışlardır. Bu çalışmada residual bloklar her 3B konvolüsyon katmanını bağlamak için kimlik eşleme yöntemini kullanırlar. Bu çalışmalara ilave olarak Cihan (2020), hiperspektral görüntüleme yöntemi ile yeni doğan sağlık durumlarını tespit etmek için komşuluk çıkarımı yöntemi ve 3B-ESA modelini kullanarak yüksek başarımlar elde etmiştir.

HSG verilerinin sınıflandırılmasında 2B-ESA kullanıldığında sadece uzamsal özellikler elde edilir. HSG verileri 3 boyutlu hacimsel veriler olduğu için hem uzamsal hem de spektral özelliklerin elde edilmesi gerekir (Roy ve ark., 2019). Bu özellikler 3 boyutlu konvolüsyon katmanları kullanılarak sağlanabilir. Ayrıca hiperspektral veriler için komşu pikseller büyük önem taşımaktadır. Bu nedenlerden dolayı makalede hem uzamsal hem de spektral özelliklerin elde edilmesi için 3B-ESA kullanılmıştır. Ayrıca HSG sınıflandırılmasında yeni bir yöntem olan komşuluk çıkarımı yöntemi kullanılarak komşu pikselleride içerecek şekilde mini küpler oluşturulmuştur. $\mathrm{Bu}$ sayede daha çok örnek kullanılarak sınıflandırılma performansının artması sağlanmıştır. Sınıflandırma performansını değerlendirmek için Indian Pines (IP), Salinas scene (SA) ve Pavia University (PU) gibi üç uzaktan algılama veriseti kullanılmıştır. Sınıflandırma işlemi sonucunda önerilen KÇ3B-ESA modeli ile yükssek başarımlar elde edilmiştir.

\section{2. Önerilen KÇ3B-ESA Modeli}

Şekil 1, HSG sınıflandırma için önerilen KÇ3B-ESA modelini göstermektedir. Giriş verilerinin boyutu WxHxD’dir. Burada W ve H uzamsal boyutu, D ise spektral boyutu ifade etmektedir. Görüntünün içerisindeki her bir piksel D boyutunda vektörlerden oluşmaktadır. Hiperspektral verilerin boyutunun büyük olmasından dolayı hem hesaplama maliyetinden kurtulmak hem de daha anlamlı bantların kullanılmasını sağlamak için giriş verilerine Temel Bileşen Analizi (TBA) uygulanmıştır. TBA uygulandıktan sonra uzamsal boyut değişmeyecek şekilde spektral boyut P'ye indirgenmiştir. Bu aşamada hacimsel verinin boyutu WxHxP'dir. Burada W ve H uzamsal boyutu ve P spektral bant sayısını ifade etmektedir.

Daha sonra Komşuluk çıkarımı yöntemini uygulamak için tüm pikseller ayrı ayrı orta nokta olacak şekilde mini küpler oluşturulmuştur. Oluşturulan bu küplere etiket değeri olarak orta noktasında bulunan gerçek etiket değerleri atanmıştır. Mini küplerin uzamsal boyutu KxK olarak belirlenmiş ve tüm P boyutundaki spektral bantlar kullanılmıştır. Komşuluk çıkarımı yönteminde amaç tüm pikselleri taramak olduğu için sıfır ekleme işlemi yapılarak ana çerçeve genişletilmiştir. KxKxP boyutunda toplamda WxH tane mini küp oluşturulmuştur.

3B verilere 3B kernel kullanarak 3B konvolüsyon işlemi uygulanmıştır. HSG verileri için önerilen modelde konvolüsyon katmanının özellik haritaları, giriş katmanındaki birden fazla bitişik bant üzerinde 3B kernel kullanılarak oluşturulmuştur. Bu durum 
spektral bilginin yakalanmasını sağlamıştır. 3 boyutlu konvolüsyon işleminin matematiksel karşılı̆̆ı aşăğda Eşitlik (1)'de gösterilmiştir.

$$
v_{i j}^{x y z}=f\left(r_{i j}+\sum_{m=0}^{M_{i}-1} \sum_{b=0}^{B_{i}-1} \sum_{h=0}^{H_{i}-1} \sum_{w=0}^{W_{i}-1} k_{i j m}^{h w b} v_{(i-1) m}^{(x+h)(y+w)(z+b)}\right)
$$

Burada $v$, özellik haritasındaki çıktı değişkenini ifade eder. $B, H, W$ verinin sırasıyla spektral ve uzamsal boyutlar boyunca filtrenin boyutunu temsil eder. $(b, h, w)$ filtre endeksleridir ve $z, x, y$ sırasıyla 2 uzamsal 1 spektral boyuta karşlık gelen özellik haritasının dizinleridir. $k$, filtre parametreleri anlamına gelir. $(i, j, m)$ sırasıyla giriş katmanı, çıkış katmanı ve özellik haritasının dizinleridir. $M$, özellik haritalarının sayısıdır, dolayısıyla $M_{i}, i$. katmanındaki özellik haritalarının sayısı anlamına gelir. $r$, bias terimidir.

ESA parametreleri $(k, r)$ genellikle dereceli alçalma optimizasyon teknikleri ile denetimli yaklaşımlar kullanılarak eğitilir (Krizhevsky ve ark., 2012). 3B-ESA kullanılarak verilerden hem uzamsal hem de spektral özellikler elde edilir. Biz 3B-ESA ve komşuluk çıkarımı yöntemini kullanarak HSG verilerinin sınıflandırılması için KÇ3B-ESA adında, özellik öğrenme modeli geliş̧irdik.

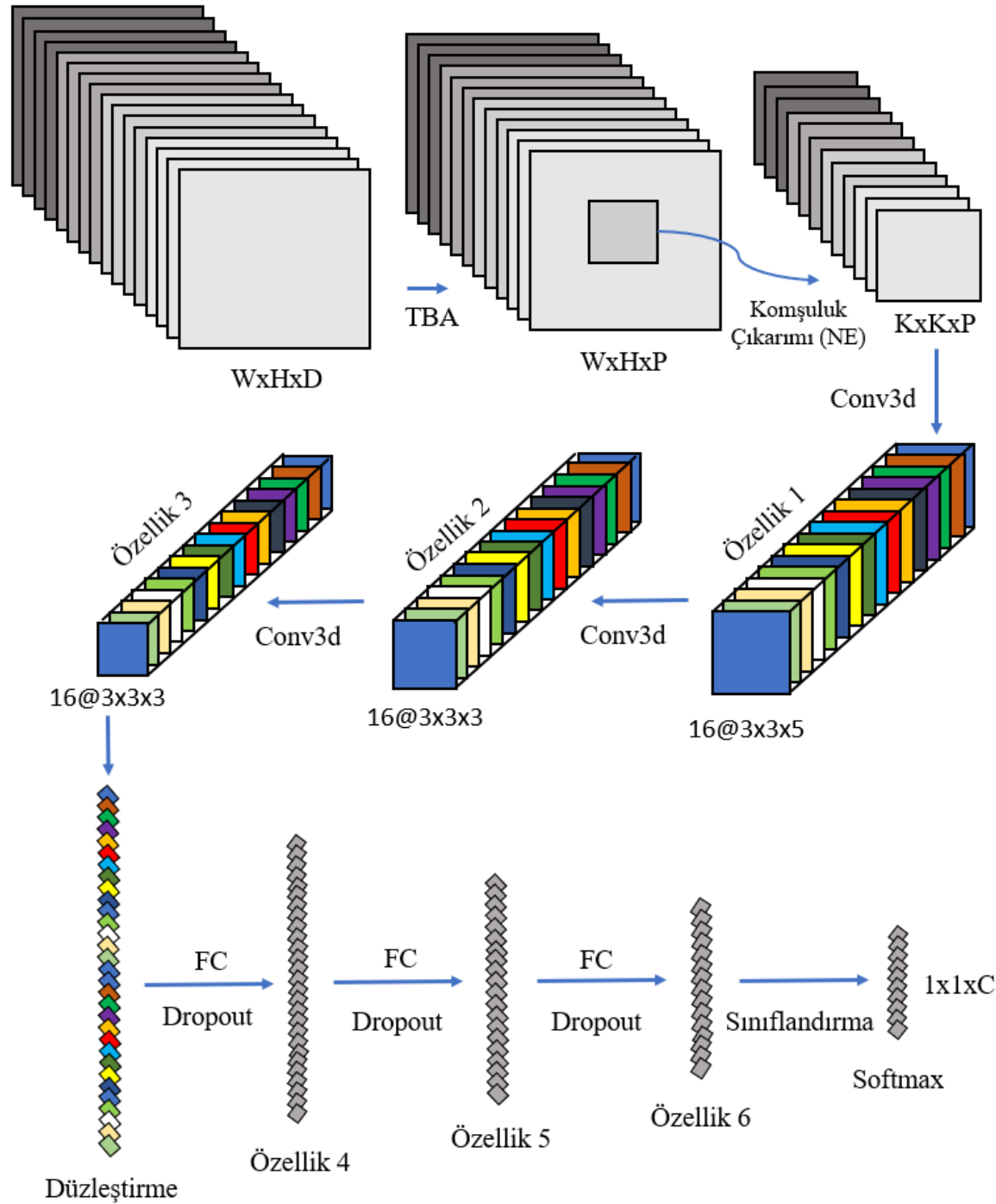

\section{Şekil 1. Önerilen KÇ3B-ESA modeli}

KÇ3B-ESA modeli 3 adet konvolüsyon katmanı içermektedir. Toplamda 3 adet konvolüsyon katmanı kullanılmıştır. İlk konvolüsyon katmanında; $3 \times 3 \times 5$ boyutunda 16 filtre, ikinci konvolüsyon katmanında; 3x3x3 boyutunda 16 filtre ve son olarak üçüncü konvolüsyon katmanında; 3×3×3 boyutunda 16 filtre kullanılarak özellik haritaları çıkarılmıştır. Daha sonra tam bağlı katmanlara (FC) girdi olarak verilmek üzere, veriler bir boyutlu tensörlere dönüştürülerek düzleştirme işlemi yapılmıştır. Düzleştirme işleminden sonra eklenen ilk ağ katmanı 128, ikincisi 64, üçüncüsü 32 nöron içermekte ve çıkış ağ katmanı sınıf sayısını belirten C nöron içermektedir. Tüm tam bağlı ağ katmanlarından sonra unutturma katmanları (dropout) kullanılmıştır. Unutturma değerleri 0.4 olarak belirlenmiştir. Tüm ağırlıklar rastgele bir şekilde başlatılmış ve softmax (Liu ve ark., 2016) aktivasyon fonksiyonu kullanarak Adam en iyileyici 
(Kingma ve $\mathrm{Ba}$, 2014) ile geri yayılım algoritması kullanılarak eğitilmiştir. Batch_size 128 olarak belirlenmiş ve ağ veri artırımı olmadan 100 epoch'ta eğitilmiştir.

\section{Deneyler ve Tartışma}

\subsection{Veriseti Tanımı ve Ăğı Eğitimi}

Bu çalışmada Indian Pines (IP), Salinas Scene (SA) ve Pavia University (PU) olarak isimlendirilen üç popüler uzaktan algılama HSG veri seti kullanılmıştır. IP veriseti $400-2500 \mathrm{~nm}$ dalga boyu aralığında $145 \times 145$ uzamsal boyutunda 224 spektral bant içermektedir. Toplam 16 sınıfa ait bitki türü bulunmaktadır (Tablo 1). SA veriseti 360-2500 nm dalga boyu aralığında $512 \times 217$ uzamsal boyutunda 224 spektral bant içermektedir. Toplam 16 farklı sınıfa ait bitki türü bulunmaktadır (Tablo 2). PU veriseti 430-860 $\mathrm{nm}$ dalga boyu aralı̆ı̆ında 610x340 uzamsal boyutunda 103 spektral bant içermektedir. Toplam 9 farklı sınıfa ait arazi türü bulunmaktadır (Tablo 3).

Tüm deneysel çalışmalar MATLAB ve Python kullanılarak NVIDIA GeForce GTX 1080 Ti GPU üzerinde, 64 GB RAM'e sahip olan bir iş istasyonunda yürütülmüştür. Öğrenme oranı başlangıçta 0.001 olarak seçilmiştir. Tüm veri setlerinde mini küplerin uzamsal boyutu 19 olarak belirlenmiştir. Spektral boyut ise tüm veri setleri için 25 olarak seçilmiştir. Sonuç olarak veri setleri için tüm mini küpler 19x19x25 boyutundadır.

Tablo 1. Indian Pines için arazi doğrulama sınıfları ve örnek sayılarl

\begin{tabular}{r|l|l}
\hline No & Sinıf & Örnek Sayısı \\
\hline $\mathbf{1}$ & Brocoli_green_weeds_1 & 2009 \\
\hline $\mathbf{2}$ & Brocoli_green_weeds_2 & 3726 \\
\hline $\mathbf{3}$ & Fallow & 1976 \\
\hline $\mathbf{4}$ & Fallow_rough_plow & 1394 \\
\hline $\mathbf{5}$ & Fallow_smooth & 2678 \\
\hline $\mathbf{6}$ & Stubble & 3959 \\
\hline $\mathbf{7}$ & Celery & 3579 \\
\hline $\mathbf{8}$ & Grapes_untrained & 11271 \\
\hline $\mathbf{9}$ & Soil_vinyard_develop & 6203 \\
\hline $\mathbf{1 0}$ & Corn_senesced_green_weeds & 3278 \\
\hline $\mathbf{1 1}$ & Lettuce_romaine_4wk & 1068 \\
\hline $\mathbf{1 2}$ & Lettuce_romaine_5wk & 1927 \\
\hline $\mathbf{1 3}$ & Lettuce_romaine_6wk & 916 \\
\hline $\mathbf{1 4}$ & Lettuce_romaine_7wk & 1070 \\
\hline $\mathbf{1 5}$ & Vinyard_untrained & 7268 \\
\hline $\mathbf{1 6}$ & Vinyard_vertical_trellis & 1807
\end{tabular}

Tablo 2. Salinas Scene için arazi doğrulama sinıfları ve örnek sayllarl

\begin{tabular}{r|l|l}
\hline No & Sinıf & Örnek SayısI \\
\hline $\mathbf{1}$ & Alfalfa & 46 \\
\hline $\mathbf{2}$ & Corn-notill & 1428 \\
\hline $\mathbf{3}$ & Corn-mintill & 830 \\
\hline $\mathbf{4}$ & Corn & 237 \\
\hline $\mathbf{5}$ & Grass-pasture & 483 \\
\hline $\mathbf{6}$ & Grass-trees & 730 \\
\hline $\mathbf{7}$ & Grass-pasture-mowed & 28 \\
\hline $\mathbf{8}$ & Hay-windrowed & 478 \\
\hline $\mathbf{9}$ & Oats & 20 \\
\hline $\mathbf{1 0}$ & Soybean-notill & 972 \\
\hline $\mathbf{1 1}$ & Soybean-mintill & 2455 \\
\hline $\mathbf{1 2}$ & Soybean-clean & 593 \\
\hline $\mathbf{1 3}$ & Wheat & 205 \\
\hline $\mathbf{1 4}$ & Woods & 1265 \\
\hline $\mathbf{1 5}$ & Buildings-Grass-Trees-Drives & 386 \\
\hline $\mathbf{1 6}$ & Stone-Steel-Towers & 93 \\
\hline
\end{tabular}

Tablo 3. Pavia University için arazi doğrulama sinıflarl ve örnek saylları

\begin{tabular}{r|l|l}
\hline No & Sinıf & Örnek Sayısı \\
\hline $\mathbf{1}$ & Asphalt & 6631 \\
\hline $\mathbf{2}$ & Meadows & 18649 \\
\hline $\mathbf{3}$ & Gravel & 2099 \\
\hline $\mathbf{4}$ & Trees & 3064 \\
$\mathbf{5}$ & Painted metal sheets & 1345 \\
\hline $\mathbf{6}$ & Bare Soil & 5029 \\
\hline $\mathbf{7}$ & Bitumen & 1330 \\
\hline $\mathbf{8}$ & Self-Blocking Bricks & 3682 \\
\hline $\mathbf{9}$ & Shadows & 947 \\
\hline
\end{tabular}

\subsection{Sınıflandırma Sonuçları}

Bu çalışmada HSG sınıflandırma performansını değerlendirmek için Genel Doğruluk (GD), Kappa Katsayısı (KC) ve Ortalama Doğruluk (OD) kullanılmıştır. GD, toplam test örnekleri arasından doğru sınıflandırılmış örneklerin sayısını temsil eder; KC, arazi doğrulama haritası ile sınıflandırma haritası arasında bilgi sağlayan bir istatistiksel ölçüm tekniğidir; ve OD, sınıflandırma doğruluklarının ortalamasına karşı1ık gelir. Önerilen KÇ3B-ESA modeli DVM (Melgani ve Bruzzone, 2004), 2B-ESA (Makantasis ve 
ark., 2015), 3B-ESA (Hamida ve ark., 2018) ve M3B-ESA (He ve ark., 2017) gibi diğer yöntemlerle karşılaştırılmıştır. Tüm veri setleri için veriler rastgele bir şekilde $\% 20$ eğitim \%80 test olacak şekilde parçalanmıştır.

Tablo 4. KÇ3B-ESA ve gelişmiş yöntemlerin IP, PU ve SA veri setleri üzerindeki sinıflandırma doğrulukları (\%)

\begin{tabular}{c|ccc|ccc|crc}
\hline \multirow{2}{*}{ Metotlar } & \multicolumn{3}{|c|}{ IP veriseti } & \multicolumn{3}{c|}{ SA veriseti } & \multicolumn{3}{c}{ PU veriseti } \\
\cline { 2 - 9 } & GD & KC & OD & GD & KC & OD & GD & KC & OD \\
\hline DVM & 85.30 & 83.10 & 79.03 & 92.95 & 92.11 & 94.60 & 94.34 & 92.50 & 92.98 \\
\hline 2B-ESA & 89.48 & 87.96 & 86.14 & 97.38 & 97.08 & 98.84 & 97.86 & 97.16 & 96.55 \\
\hline 3B-ESA & 91.10 & 89.98 & 91.58 & 93.96 & 93.32 & 97.01 & 96.53 & 95.51 & 97.57 \\
\hline M3B-ESA & 95.32 & 94.70 & 96.41 & 94.79 & 94.20 & 96.25 & 95.76 & 94.50 & 95.08 \\
\hline KÇ3B-ESA & 99.10 & 98.97 & 96.23 & 100 & 100 & 100 & 99.90 & 99.87 & 99.67 \\
\hline
\end{tabular}

Tablo 4 farklı metotlar için sınıflandırma sonuçlarını göstermektedir. Tabloda görüldüğü gibi tasarlanan KÇ3B-ESA modeli diğer yöntemlere göre daha iyi başarılar elde etmiştir. Bunun sebebi 3B konvolüsyon katmanlarının kullanılması ile hem spektral hem de uzamsal özelliklerinin elde edilmesinin yanı sıra komşuluk çıkarımı yöntemi ile örnekleme sayısının artırılarak daha fazla verinin kullanılmasıdır. SA veriseti incelendiğinde 2B-ESA yönteminde 3B-ESA yöntemine göre daha iyi sonuçlar elde edildiği görülmektedir. Bunun sebebi bu veri setinde muhtemelen birçok spektral bantta benzer özelliklerin olduğudur. Bizim tasarladığımız KÇ3B-ESA modeli ile bu veri setinde tüm sınıflar doğru tahmin edilerek performans kriterleri \%100 oranında elde edilmiştir.

Şekil 2'de IP veri setine ait şekiller verilmiştir. Şekil 2(a)'da arazi doğrulama haritası (gerçek sınıfların bulunduğu harita), Şekil 2(b)'de tahmin edilen sınıflandırma haritası ve Şekil 2(c-d)' de ise eğitim ve doğrulama setleri için 100 epoch'ta doğruluk ve kayıp grafikleri verilmiştir. Bu veriseti için model, 100 epoch sonunda yaklaşık olarak \%98 oranında eğitilmiştir. Ayrıca Şekil 3'te SA, Şekil 4 'te ise PU veri seti için oluşturulmuş doğrulama haritası, tahmin edilen sınıflandırma haritası, eğitim doğruluğu ve eğitim kaybı grafikleri verilmiştir. Ayrıca bu iki veriseti için model, yaklaşık 20. epoch’tan sonra tam olarak eğitilmiştir. Tüm bu şekiller incelendiğinde önerilen KÇ3B-ESA modeli ile yüksek başarımlara ulaşıldığı açıkça görülmektedir.

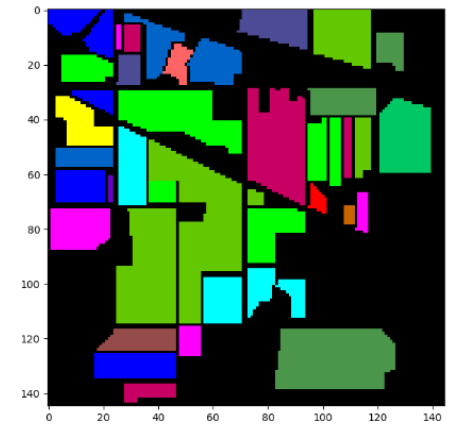

(a)

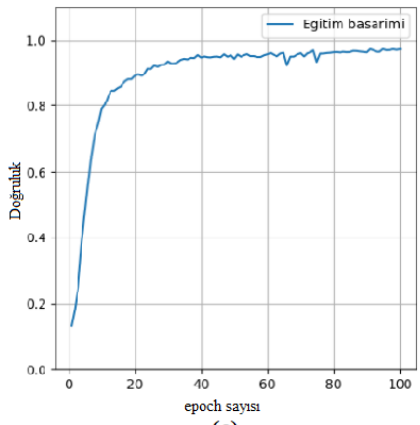

(c)

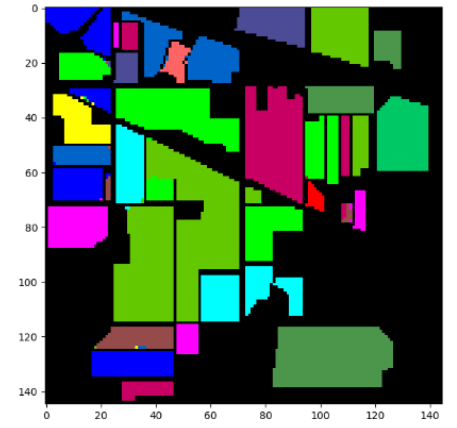

(b)

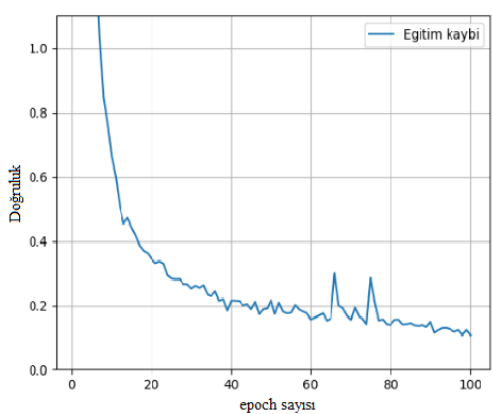

(d)

Şekil 2. Indian Pines, (a) arazi doğrulama haritası (b) tahmin edilen sınıflandırma haritası (c) eğitim başarımı (d) eğitim kaybı 


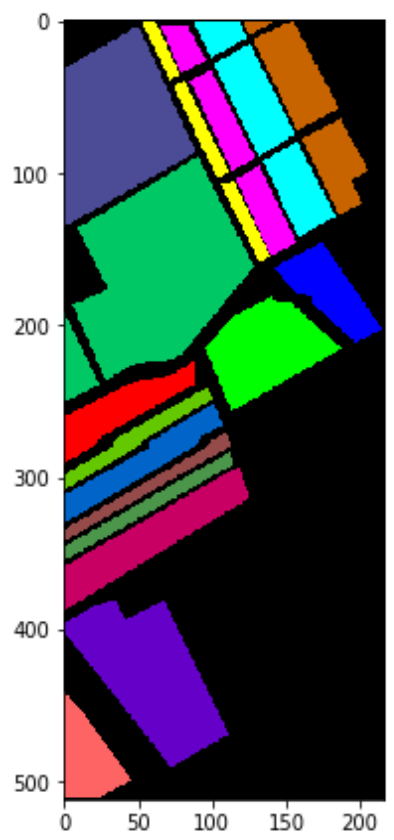

(a)

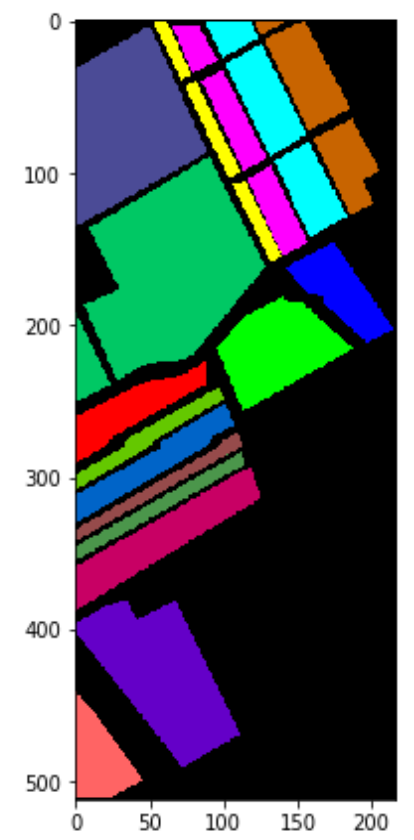

(b)

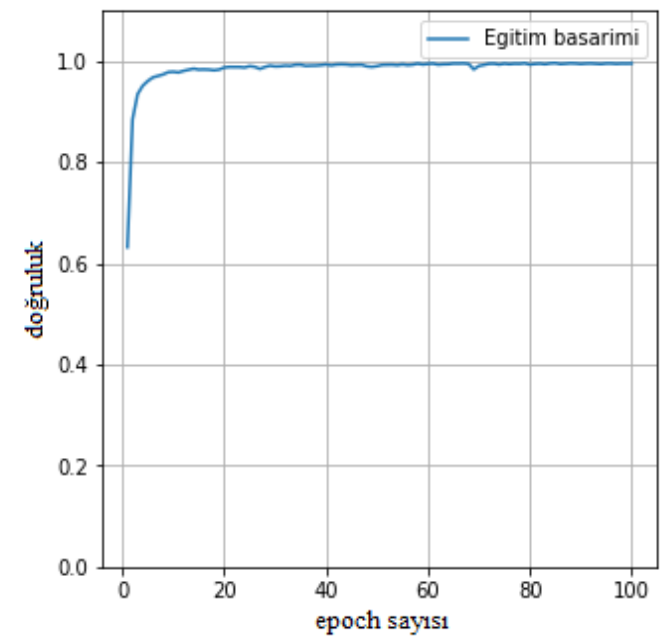

(c)

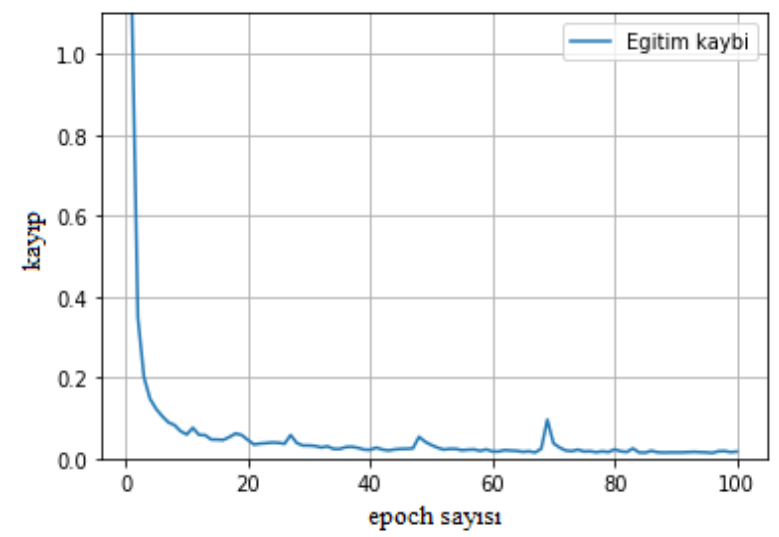

(d)

Şekil 3. Salinas Scene, (a) arazi doğrulama haritası (b) tahmin edilen sınıflandırma haritası (c) eğitim bașarımı (d) eğitim kaybı

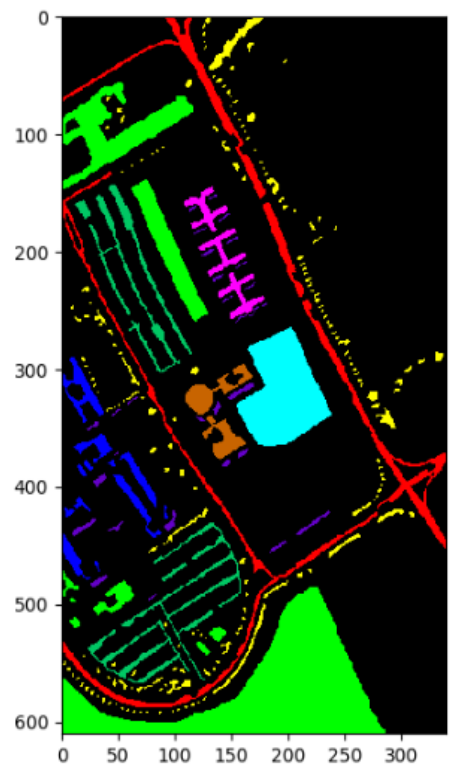

(a)

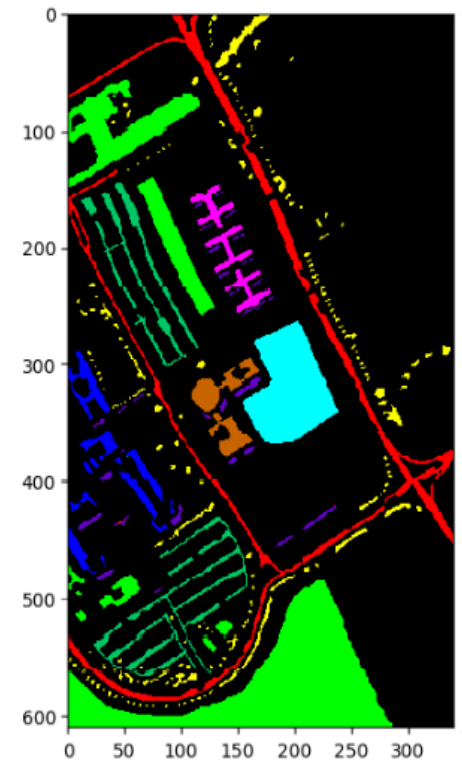

(b)

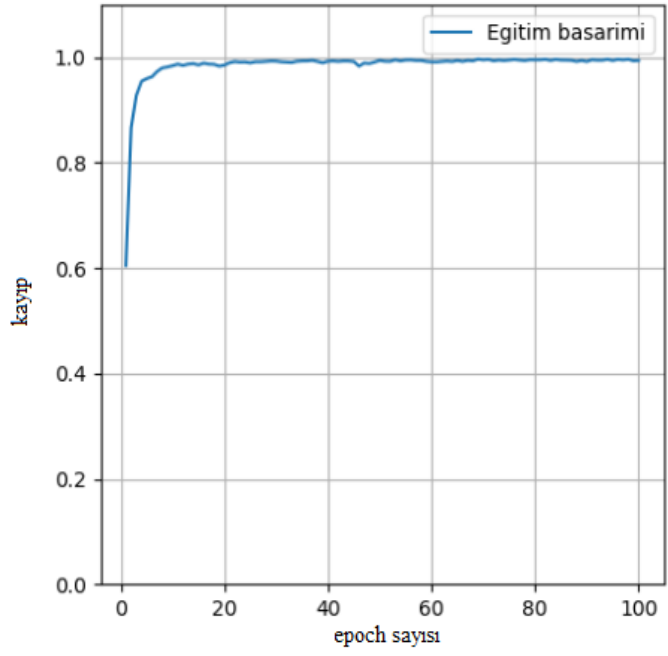

(c)

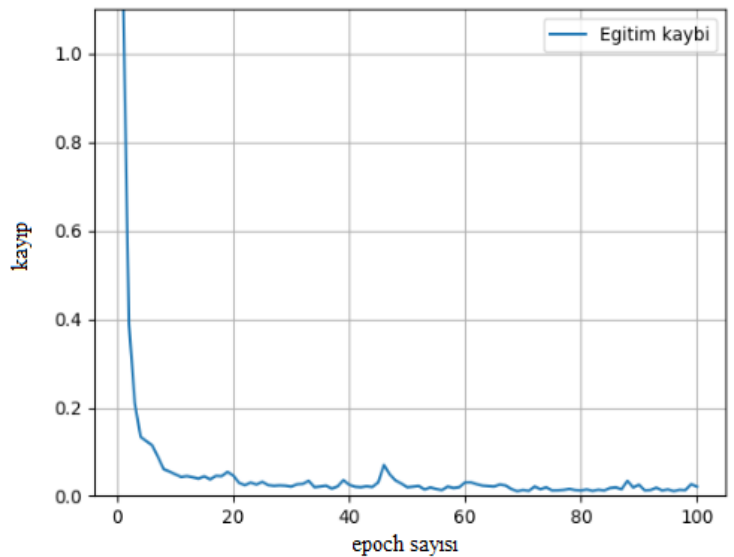

(d)

Şekil 4. Pavia University, (a) arazi doğrulama haritasl (b) tahmin edilen sinıflandırma haritası (c) eğitim bașarımı (d) eğitim kaybı 


\section{Sonuç}

Bu makalede HSG sınıflandırması için komşuluk çıkarımı ve 3B-ESA kullanılarak yeni bir model tanıtılmıştır. Önerilen 3B-ESA modeli ile hem uzamsal hem de spektral özelliklerin çıkarılması hedeflenmiștir. Ayrıca KÇ yöntemi ile tüm pikselleri tarayacak şekilde mini küpler oluşturularak örnek sayısının artırılması işlemi gerçekleştirilmiştir. Sonuç olarak önerilen KÇ3B-ESA modeli ile üç farklı değerlendirme veriseti kullanılarak sınıflandırma işlemi gerçekleştirilmiştir. Ede edilen sınıflandırma sonuçları diğer gelişmiş yöntemlerle kıyaslanarak önerilen modelin başarısı ispatlanmıştır. Ayrıca, çalışmada çok az eğitim verisi kullanılmasına rağmen yüksek doğrulukta başarımlar elde edilmiştir.

\section{Kaynakça}

Camps-Valls, G., \& Bruzzone, L. (2005). Kernel-based methods for hyperspectral image classification. IEEE Transactions on Geoscience and Remote Sensing, 43(6), 1351-1362.

Chu, W., \& Cai, D. (2018). Deep feature based contextual model for object detection. Neurocomputing, 275, 1035-1042.

Cihan, M. (2020). Hiperspektral Görüntüleme Yöntemi Kullanılarak Yenidoğan Sağlık Durumlarının Derin Öğrenme Metotları ile Sınıflandırılması. Yayımlanmış Yüksek Lisans Tezi, Konya Teknik Üniversitesi, Konya.

Gidaris, S., \& Komodakis, N. (2015). Object detection via a multi-region and semantic segmentation-aware cnn model. In Proceedings of the IEEE international conference on computer vision, 1134-1142.

Ham, J., Chen, Y., Crawford, M. M., \& Ghosh, J. (2005). Investigation of the random forest framework for classification of hyperspectral data. IEEE Transactions on Geoscience and Remote Sensing, 43(3), 492-501.

Hamida, A. B., Benoit, A., Lambert, P., \& Amar, C. B. (2018). 3-D deep learning approach for remote sensing image classification. IEEE Transactions on geoscience and remote sensing, 56(8), 4420-4434.

He, M., Li, B., \& Chen, H. (2017). Multi-scale 3D deep convolutional neural network for hyperspectral image classification. In 2017 IEEE International Conference on Image Processing (ICIP), 3904-3908.

Huang, K., Li, S., Kang, X., \& Fang, L. (2016). Spectral-spatial hyperspectral image classification based on KNN. Sensing and Imaging, 17(1), 1.

Kingma, D. P., \& Ba, J. (2014). Adam: A method for stochastic optimization. ArXiv preprint arXiv:1412.6980.

Krizhevsky, A., Sutskever, I., \& Hinton, G. E. (2012). Imagenet classification with deep convolutional neural networks. In Advances in neural information processing systems, 1097-1105.

Lawrence, R. L., Wood, S. D., \& Sheley, R. L. (2006). Mapping invasive plants using hyperspectral imagery and Breiman Cutler classifications (RandomForest). Remote Sensing of Environment, 100(3), 356-362.

Lee, H., \& Kwon, H. (2016). Contextual deep CNN based hyperspectral classification. In 2016 IEEE International Geoscience and Remote Sensing Symposium (IGARSS), 3322-3325.

Li, Y., Xie, W., \& Li, H. (2017). Hyperspectral image reconstruction by deep convolutional neural network for classification. Pattern Recognition, 63, 371-383.

Liu, F., Shen, C., \& Lin, G. (2015). Deep convolutional neural fields for depth estimation from a single image. In Proceedings of the IEEE conference on computer vision and pattern recognition, 5162-5170.

Liu, W., Wen, Y., Yu, Z., \& Yang, M. (2016, June). Large-margin softmax loss for convolutional neural networks. In ICML, 2(3), 7.

Ma, L., Crawford, M. M., \& Tian, J. (2010). Local manifold learning-based k-nearest-neighbor for hyperspectral image classification. IEEE Transactions on Geoscience and Remote Sensing, 48(11), 4099-4109.

Makantasis, K., Karantzalos, K., Doulamis, A., \& Doulamis, N. (2015). Deep supervised learning for hyperspectral data classification through convolutional neural networks. In 2015 IEEE International Geoscience and Remote Sensing Symposium (IGARSS), 4959-4962.

Melgani, F., \& Bruzzone, L. (2004). Classification of hyperspectral remote sensing images with support vector machines. IEEE Transactions on geoscience and remote sensing, 42(8), 1778-1790.

Ren, S., He, K., Girshick, R., \& Sun, J. (2015). Faster r-cnn: Towards real-time object detection with region proposal networks. In Advances in neural information processing systems, 91-99.

Roy, S. K., Krishna, G., Dubey, S. R., \& Chaudhuri, B. B. (2019). HybridSN: Exploring 3-D-2-D CNN feature hierarchy for hyperspectral image classification. IEEE Geoscience and Remote Sensing Letters, 17(2), $277-281$.

Saba, T., Khan, M. A., Rehman, A., \& Marie-Sainte, S. L. (2019). Region extraction and classification of skin cancer: A heterogeneous framework of deep CNN features fusion and reduction. Journal of medical systems, 43(9), 289.

Wang, J., Yang, Y., Mao, J., Huang, Z., Huang, C., \& Xu, W. (2016). Cnn-rnn: A unified framework for multi-label image classification. In Proceedings of the IEEE conference on computer vision and pattern recognition, 2285-2294.

Zhao, W., \& Du, S. (2016). Spectral-spatial feature extraction for hyperspectral image classification: A dimension reduction and deep learning approach. IEEE Transactions on Geoscience and Remote Sensing, 54(8), 4544-4554.

Zhong, Z., Li, J., Luo, Z., \& Chapman, M. (2017). Spectral-spatial residual network for hyperspectral image classification: A 3-D deep learning framework. IEEE Transactions on Geoscience and Remote Sensing, 56(2), 847-858. 